\title{
THORIA EHANCEMENT OF NUCLEAR REACTOR SAFETY
}

\author{
${ }^{1}$ Barbara Szpunar and ${ }^{2}$ Jerzy Szpunar \\ ${ }^{1}$ Department of Physics and Engineering Physics, University of Saskatchewan, Saskatoon, Canada \\ ${ }^{2}$ Department of Mechanical Engineering, University of Saskatchewan, Saskatoon, Canada
}

Received 2013-10-25; Revised 2013-12-20; Accepted 2013-12-20

\begin{abstract}
The recent nuclear accident in Fukushima demonstrates that a nuclear mishap should be viewed using interdisciplinary tools. For example, the urania fuel melts not only because of enhanced neutron flux but also because its thermal conductivity degrades when it oxidizes. Here we present the application of first principles calculations to evaluate the structural, mechanical and thermal properties of traditional urania fuel and new, inherently safer thoria fuel. Knowledge of the lattice constants for higher oxidation state oxides of uranium is important in nuclear safety analysis, since the formation of $\mathrm{U}_{3} \mathrm{O}_{8}$ in a defective fuel element can cause cracking or split the fuel sheath after disposal, due to a net 32-36\% volume increase. This contrasts with thoria fuel where such oxidation does not occur during accident. The comparison between these two fuels is provided with respect to reactor safety. In an exemplary simulation we show that replacement of urania fuel by thoria prevents central melting in the fuel rod.
\end{abstract}

Keywords: Thoria, Urania, Nuclear Reactor Safety, Simulations

\section{INTRODUCTION}

The recent tragic accident in Fukushima clearly illustrates the risks associated with the present design of reactors based on uranium oxide fuel and justifies the research towards a safer fuel. Traditionally, safety protocols focus on reactor physics to evaluate the behavior of a nuclear reactor. However real nuclear accidents (like the recent reactor failure in Fukushima) demonstrate that a nuclear mishap should be viewed using interdisciplinary tools.

For example the fuel melts not only because of enhanced neutron flux but also because its thermal conductivity degrades when fuel oxidation takes place (Lewis et al., 2002). Another study (Szpunar et al., 2001) shows that urania fuel does not start to oxidize until the protective Zircaloy cladding is fully oxidized. However during the oxidation of Zircalloy cladding hydrogen is produced $\left(\mathrm{Zr}+2 \mathrm{HO}_{2} \rightarrow \mathrm{ZrO}_{2}+2 \mathrm{H}_{2}\right)$ and it may explode as happened during the Fukushima accident.

Fresh urania fuel has a cubic crystal structure (Fm $\overline{3} \mathrm{~m}$ symmetry observed experimentally (Wyckoff, 1963).
$\mathrm{UO}_{2}$ has a complex magnetic structure (noncollinear triple-k antiferromagnetic ordering (Wilkins et al., 2006)), which is stable only up to $\mathrm{T}_{\mathrm{N}}=30.8 \mathrm{~K}$ (Frazer et al., 1965), whereas the experimentally available data on structural and mechanical properties are measured in the paramagnetic state. In the paramagnetic state there is no long-range order, but, because $5 \mathrm{f}$ electrons are strongly localized, the local moments (1.74 $\mu_{\beta}$ (Faber et al., 1976; Lander, 1980)) should not be affected much by magnetic ordering.

The most stable uranium oxide is $\mathrm{U}_{3} \mathrm{O}_{8}$. with the orthorhombic pseudo-hexagonal phase $(\mathrm{C} 2 \mathrm{~mm})$ at room temperature and hexagonal phase $(\mathrm{P} \overline{6} 2 \mathrm{~m})$ that is stable above $400^{\circ} \mathrm{C}$ (Loopstra, 1970). Interestingly when urania oxidizes initially to $\mathrm{U}_{4} \mathrm{O}_{9}$ a small shrinking of volume occurs as discussed in (Szpunar and Szpunar, 2004) and is attributed to the reduction of the magnetic moment on uranium. However $\mathrm{U}_{3} \mathrm{O}_{8}$ has higher volume per $\mathrm{U}$ atom than $\mathrm{UO}_{2}$ and the respective relative linear expansion of urania $v=1.36$ (McEachern and Taylor, 1998) and $v=$ 1.32 (Iglesias et al., 1989) was observed experimentally during oxidation to $\mathrm{U}_{3} \mathrm{O}_{8}$. The created stress (Szpunar et al., 
2013, Szpunar and Szpunar, 2013b) during formation of $\mathrm{U}_{3} \mathrm{O}_{8}$ affects not only the safety of the nuclear reactor but also the fuel storage facilities as cracking and fragmentation may occur. The oxidation and reduction cycle (OREOX) can also break the fuel sheath (Sullivan and Cox, 1995) due to increasing stress caused by the expansion of oxidized fuel $\left(\mathrm{U}_{3} \mathrm{O}_{8}\right)$. This process is also used to produce the powdered uranium oxide for fuel pellet production.

Thoria fuel presents an attractive alternative to traditional urania fuel. It is widely discussed that thoria is not only four times more abundant but also that nuclear reactors based on thorium would be safe, with the risk of reactor core melt-down eliminated due not only to higher thermal conductivity but also a much higher fuel melting temperature: $3651 \pm 17 \mathrm{~K}$ (Ronchi and Hiernaut, 1996) versus $3120 \pm 30 \mathrm{~K}$ (Adamson et al., 1985) for urania. However it is seldom discussed that thoria does not oxidize to the higher oxidation states like urania. Therefore it has the further benefit that cracking and fragmentation would not occur as happens with urania. Additionally its thermal conductivity does not deteriorate due to oxidation as with urania, (Lewis et al., 2002) and these characteristics will be explored here.

\subsection{Application of First Principles Calculations}

\subsubsection{Uranium Oxides}

The commonly used Density Functional Theory (DFT) (Kohn and Sham, 1965) within the Local-Density Approximation (LDA) (Ceperley and Alder, 1980) predicts that urania is metallic, while experiment has observed it to be an insulator with a $2.1 \mathrm{eV}$ band gap (Baer and Schoenes, 1980). To address shortcomings of DFT that underestimate the influence of the strong onsite Coulomb repulsion between the strongly correlated 5f electrons, in our work the Hubbard U correction (DFT+U scheme (Cococcioni and Gironcoli, 2005)) is used. CASTEP (Segall et al., 2002) calculations with LDA and LDA+U for the effective $U$ value of $2 \mathrm{eV}$ and $3.5 \mathrm{eV}$ were presented (Szpunar, 2012a). The latter value reproduces the best experimental optical band gap of 2.1 eV (Baer and Schoenes, 1980). The shift in energy and the width of the created gap are proportional to the $U$ value, as expected. We observed exactly the same energy shifts and band gaps for two uranium atoms in antiferromagnetic urania with antiparallel magnetic moment orientation (Szpunar, 2013).

\section{2. $\mathrm{U}_{3} \mathrm{O}_{8}$}

There are about $67 \%$ more oxygen atoms per uranium atom in $\mathrm{U}_{3} \mathrm{O}_{8}$ than in $\mathrm{UO}_{2}$. In the $\mathrm{P} 2 \mathrm{~m}$ structure all uranium sites are equivalent; therefore when assuming the same symmetry for the electronic structure the simple valency consideration leads to 5.33 noninteger valency of ions (1.33 valence increase from quadrivalent ionic state in $\left.\mathrm{UO}_{2}\right)$. Therefore as discussed previously (Szpunar et al., 2013; Szpunar and Szpunar, 2013b) there is no band gap formed for the $\mathrm{U}_{3} \mathrm{O}_{8}$ compound unless equivalency symmetry restrictions are removed and it is allowed for uranium ions to have different valency (e.g., from simple model: Two hexavalent and one tetravalent state or two hexavalent and one pentavalent state as indicated in (Yun et al., 2011)). Therefore the band gap value: $2.17 \mathrm{eV}$ comparable to the observed value in urania (Baer and Schoenes, 1980) has been found (Szpunar et al., 2013; Szpunar and Szpunar, $2013 \mathrm{~b})$ in orthorhombic $(\mathrm{C} 2 \mathrm{~mm}) \mathrm{U}_{3} \mathrm{O}_{8}$ for $\mathrm{U}$ value equal to $6 \mathrm{eV}$ (note: Proposed before in (Geng et al., 2007) for urania). However this band gap value depends on the value of Hubbard U. There are no experimental data available about this band gap width.

The most important prediction of the first principles simulation is that there is an increase in the volume of between $35-39 \%$ per uranium atom (independent on the used functional) during transformation from $\mathrm{UO}_{2}$ to $\mathrm{U}_{3} \mathrm{O}_{8}$ (Szpunar et al., 2013; Szpunar and Szpunar, 2013b) and therefore cracking and fragmentation of urania fuel occurs during oxidation (Szpunar, 2013) as also discussed above.

\subsection{Thoria Versus Urania Fuel}

The crystal structure of thoria is cubic $\mathrm{Fm} \overline{3} \mathrm{~m}$, like urania, but it is nonmagnetic. In contrast to urania, thoria is predicted to be an insulator within the LDA (or GGA) scheme, although the band gap's values are underestimated. However as discussed previously (Szpunar et al., 2013) for thoria to represent correctly the wide band gap of $6 \mathrm{eV}$ (Sviridova and Suikovskaya, 1967), the 5f electrons are only pushed up sufficiently when the B3LYP functional (Becke, 1993) is used. This leads to a larger band gap $(6.9 \mathrm{eV})$, in better agreement with experiment $(6 \mathrm{eV})$ than value $(4.7 \mathrm{eV})$ calculated using the LDA+U (6 eV) scheme (Szpunar et al., 2013). In our recent research we have studied the mechanical, structural, electronic and optical properties of thoria (Szpunar et al., 2013, Szpunar and Szpunar, 2013b). In the following two sections we summarize our preliminary studies using DFT to compare thermal expansion, melting temperatures and minimal thermal conductivity of thoria and urania as presented in more details (Szpunar, 2013). 


\subsection{Melting Temperature}

Recent research (Sanati et al., 2011) demonstrated that empirical correlation (Fine et al., 1984) between $\mathrm{C}_{11}$ elastic constant and melting temperature $\left(\mathrm{T}_{\mathrm{m}}\right)$ furnishes the latest in good agreement with experiment. Table 1 shows the evaluated melting temperature for urania and thoria. We used the previously calculated (by us) $\mathrm{C}_{11}$ using various functionals (Szpunar et al., 2013) and the empirical correlation (Fine et al., 1984) Equation (1):

$$
\mathrm{T}_{\mathrm{m}}[\mathrm{K}]=553+5.91 \mathrm{C}_{11}[\mathrm{GPa}]
$$

We note that this relationship was tested for various cubic metals and compounds and agreement was within $300 \mathrm{~K}$.

The experimental value of the melting temperature of urania $(3120 \pm 30 \mathrm{~K}$ (Adamson et al., 1985)) is lower than that evaluated as the most accurate (Bakker et al., 1997) value at exact stoichiometry (3651 $\pm 17 \mathrm{~K}$ (Ronchi and Hiernaut, 1996)) and other evaluated melting temperatures between 3323 and $3663 \mathrm{~K}$ (Bakker et al., 1997). However as Table 1 shows the estimated melting temperature of thoria is lower than urania and it is underestimated by more than $300 \mathrm{~K}$ while for urania agreement is good for most functionals (except of PBE).

\subsection{Minimal Thermal Conductivity}

The phonon contribution to thermal conductivity decreases with temperature (e.g., for urania Ref. (Lewis et al., 2002)) and it is of interest for reactor safety analysis to estimate the minimum thermal conductivity that according to (Cahill et al., 1992) is proportional to sound velocity v Equation (2):

$$
\mathrm{v}=\sqrt{\mathrm{B} / \rho}
$$

where, B is bulk modulus and $\rho$ is density. Since thoria and urania have the same structure, the ratio of the minimal thermal conductivity is equal to the ratio of sound velocities and it can be expressed in the previously evaluated (e.g., in (Szpunar et al., 2013; Szpunar and Szpunar, 2013a)) bulk moduli (B), lattice constants (a) as Equation (3):

$$
\begin{aligned}
& \frac{\kappa_{\mathrm{min}}^{\mathrm{ThO}_{2}}}{\kappa_{\min }^{\mathrm{UO}_{2}}}=\frac{\mathrm{v}_{\mathrm{s}}^{\mathrm{ThO}_{2}}}{\mathrm{v}_{\mathrm{s}}^{\mathrm{UO}_{2}}}=\sqrt{\frac{\mathrm{B}_{\mathrm{ThO}_{2}}\left(\mathrm{~A}_{\mathrm{U}}+2 \mathrm{~A}_{\mathrm{O}}\right) \mathrm{a}_{\mathrm{ThO}_{2}}^{3}}{\mathrm{~B}_{\mathrm{UO}_{2}}\left(\mathrm{~A}_{\mathrm{Th}}+2 \mathrm{~A}_{\mathrm{O}}\right) \mathrm{a}_{\mathrm{UO}_{2}}^{3}}} \\
& =\sqrt{\frac{270 \mathrm{~B}_{\mathrm{ThO}_{2}} \mathrm{a}_{\mathrm{ThO}_{2}}^{3}}{264 \mathrm{~B}_{\mathrm{UO}_{2}} \mathrm{a}_{\mathrm{UO}_{2}}^{3}}}
\end{aligned}
$$

where, $\mathrm{A}_{\text {element }}$ are atomic masses of the indicated atoms. The calculated ratio of the thermal conductivity of $\mathrm{ThO}_{2}$ versus $\mathrm{UO}_{2}$ (phonon contribution) at $1800 \mathrm{~K}$ as used in (Bakker et al., 1997; Lewis et al., 2002) is 1.02 and compares well with the estimated values in Table 2 for various functionals (LDA (Ceperley and Alder, 1980), WC (Wu and Cohen, 2006), LDA+U (Cococcioni and Gironcoli, 2005) (urania), B3LYP (Becke, 1993) (thoria) and experimental data).

\subsection{Thermal Conductivity of Urania and Thoria}

\subsubsection{Urania}

In order to determine the temperature distribution in uranium oxides, the thermal conductivity of the fuel must be known. There are three possible mechanisms of the heat conduction in urania: Lattice vibration (phonons), electron-hole movement (polarons), radiative contributions (photons). The phonon contribution to the thermal conductivity of urania $\mathrm{k}_{\mathrm{ph}}$ can be represented by the expression (Lucuta et al., 1996; Ellis et al., 2000):

$$
\mathrm{k}_{\mathrm{ph}}=\frac{1}{\mathrm{~A}(\mathrm{x})+\mathrm{B}(\mathrm{x}) \mathrm{T}} \quad \mathrm{W} \mathrm{m}^{-1} \mathrm{~K}^{-1}
$$

where according to Lucuta et al. (1996):

$$
\begin{aligned}
& A(x)=0.0257+3.336 x \\
& B(x)=0.0002206-0.000685 x
\end{aligned}
$$

Or as proposed by Ellis et al. (2000):

$$
\begin{aligned}
& \mathrm{A}(\mathrm{x})=0.014-0.010763 \sqrt{\mathrm{x}}-2.3814 \mathrm{x} \\
& +12.81986(\sqrt{\mathrm{x}})^{3}-5 \mathrm{x}^{3} \\
& \mathrm{~B}(\mathrm{x})=0.0002218+0.0002562 \sqrt{\mathrm{x}}-0.00064 \mathrm{x} \\
& -0.0036764(\sqrt{\mathrm{x}})^{3}+0.0173 \mathrm{x}^{3}
\end{aligned}
$$

The parameter $\mathrm{A}$ in Equation $4 \mathrm{c}$ has been slightly modified from the original analysis in order to predict the central melting that was observed in the lower part of the fuel rod in experiment FFO-103 at a linear power rating of $52 \mathrm{~kW} / \mathrm{m}$ (Lewis et al., 2002).

At high temperatures the phonons' contribution to thermal conductivity becomes less important while the reverse is true for the radiative contribution. The radiative term in the current analysis is based on the previously proposed contribution (Hyland, 1983; Reid et al., 1997):

$\mathrm{k}_{\mathrm{rad}}=7.59375 \times 10^{-7}\left(\alpha_{\mathrm{R}}(\mathrm{T})\right)^{-1} \mathrm{~T}^{3}$ 
Table 1. C11 elastic constants and melting temperature evaluated using Equation 1

\begin{tabular}{llll}
\hline Compound & Functionals & $\mathrm{C}_{11}[\mathrm{GPa}]$ & $\mathrm{T}_{\mathrm{m}}[\mathrm{K}]$ \\
\hline $\mathrm{ThO}_{2}$ & LDA (Ceperley and Alder, 1980) & 385.0 & 2828.350 \\
& PBE (Perdew et al., 1966) & 351.9 & 2632.729 \\
& PBEsol (Perdew et al., 2008) & 370.9 & 2745.019 \\
& WC (Wu and Cohen, 2006) & 370.6 & 2743.246 \\
& B3LYP (Becke, 1993) & 373.1 & 2758.021 \\
& LDA+U & 337.0 & 2544.670 \\
& Exp. & 377.0 (Clausen et al., 1987) & 2781.070 \\
$\mathrm{UO}_{2}(\mathrm{AF})$ & Exp. & 367.0 (Macedo et al., 1964) & 2721.970 \\
$\mathrm{UO}_{2}$ & LDA+U & 389.2 & 2853.172 \\
& LDA+U & 370.5 & 2742.655 \\
& LDA (Ceperley and Alder, 1980) & 411.9 & 2987.329 \\
& PBE (Perdew et al., 1966) & 318.2 & 2433.562 \\
& WC & 398.0 & 2905.180 \\
& Exp. & 389.0 (Fritz, 1976) & 2851.990 \\
\hline
\end{tabular}

Table 2. The ratio of the minimum of the thermal conductivity of thoria versus urania as evaluated using Equation 3

\begin{tabular}{llllll}
\hline Method & $\mathrm{B}_{\mathrm{ThO} 2}$ & $\begin{array}{l}\mathrm{a}_{\mathrm{ThO} 2} \\
{[\mathrm{~nm}]}\end{array}$ & $\begin{array}{l}\mathrm{B}_{\mathrm{UO} 2} \\
{[\mathrm{GPa}]}\end{array}$ & $\begin{array}{l}\mathrm{a}_{\mathrm{UO} 2} \\
{[\mathrm{~nm}]}\end{array}$ & $\begin{array}{l}\mathrm{K}_{\min } \\
\left(\mathrm{ThO}_{2} / \mathrm{UO}_{2}\right)\end{array}$ \\
\hline LDA & 213.4 & 0.553 & 229.1 & 0.53300 & 1.03 \\
GGA/WC & 203.0 & 0.556 & 221.7 & 0.53500 & 1.02 \\
LDA+U/B3LYP & 196.2 & 0.559 & 212.5 & 0.54600 & 1.01 \\
Experiment & 208 (av.) & 0.560 & 208.9 & 0.54582 & 1.05 \\
& (Clausen et al., 1987; & (Mathews et al., & (Fritz, 1976) & (Wyckoff, 1963) & \\
& Macedo et al., 1964) & 2000) & & & \\
\hline
\end{tabular}

Where:

$\alpha_{\mathrm{R}}(\mathrm{T})=\mathrm{C}_{1} \exp \left(\mathrm{C}_{2} \times \mathrm{T}\right)$

with $\mathrm{C}_{1}$ equal to $8750 \mathrm{~m}^{-1}$ and $\mathrm{C}_{2}$ having a value of $7.5971 \times 10^{-4} \mathrm{~K}^{-1}$.

Additionally, at high temperature for urania fuel there is a significant electron-hole: (Polaron) transport contribution to the thermal conductivity (Reid et al., 1997; Lewis et al., 2002):

$$
\mathrm{k}_{\mathrm{e}}=\left(\frac{\mathrm{k}_{\mathrm{B}}}{\mathrm{e}}\right)^{2} \mathrm{~T} \frac{\sigma_{\mathrm{e}} \sigma_{\mathrm{h}}}{\sigma_{\mathrm{e}}+\sigma_{\mathrm{h}}}\left(\frac{\Delta \mathrm{U}}{\mathrm{k}_{\mathrm{B}} \mathrm{T}}\right)^{2}
$$

where, $k_{B}$ is the Boltzman constant, e is the electron charge, $\sigma_{\mathrm{e}}$ is partial dc electric conductivity due to electron transport, $\sigma_{\mathrm{h}}$ is the partial dc electric conductivity due to the hole transport and $\Delta \mathrm{U}$ is the Mott-Hubbard energy gap. According to (Reid et al., 1997) the above expression for a given temperature and stoichiometric deviation $(\mathrm{x})$ leads to the formula:

$$
\mathrm{k}_{\mathrm{e}}=\mathrm{C}_{\sigma}\left(\frac{\Delta \mathrm{U}}{\mathrm{k}_{\mathrm{B}} \mathrm{T}}\right)^{2} \frac{\mathrm{np}(1-\mathrm{n}-\mathrm{p})}{\mathrm{n}+\mathrm{p}} \mathrm{e}^{-\Delta \mathrm{E} /\left(\mathrm{k}_{\mathrm{B}} \mathrm{T}\right)}
$$

where, $\mathrm{C}_{\sigma}$ is a constant equal to $3.71 \mathrm{Wm}^{-1} \mathrm{~K}^{-1}, \Delta \mathrm{U}$ is the Mott-Hubbard energy gap equal to $4.33 \times 10^{-19} \mathrm{~J}, \mathrm{k}_{\mathrm{B}}$ is the Boltzman constant, $\Delta \mathrm{E}$ is the electron mobility activation energy equal to $4.81 \times 10^{-20} \mathrm{~J}$. The variables $\mathrm{p}$ and $\mathrm{n}$ are calculated from the conditions of electroneutrality and thermodynamic equilibrium. The molar electron (n) and hole (p) concentration for a given stoichiometric deviation $(\mathrm{x})$ are represented by equations:

$p=x+\frac{-2 \gamma+\sqrt{x^{2}(1-4 \gamma)+\gamma}}{1-4 \gamma}$

$n=p-2 x$

Where:

$$
\gamma=\exp \left(-\Delta \mathrm{F} /\left(\mathrm{k}_{\mathrm{B}} \times \mathrm{T}\right)\right)
$$

$\Delta \mathrm{F}=\Delta \mathrm{U}-\mathrm{T} \Delta \mathrm{S}$ and $\Delta \mathrm{S}=2.62 \times 10^{-23} \mathrm{~J} / \mathrm{K}$

Recently Fink (2000) made recommendations for the thermal conductivity correlation of stoichiometric $\mathrm{UO}_{2}$ and in the recent review (Carbajo et al., 2001) correlation was provided for oxidized urania. In these proposed correlations the high temperature contribution 
is fitted by a single (ambipolar) term without separation of the radiative contribution. In contrast to the electronic contribution's dependence on a stoichiometry deviation, as presented here, the recommended (Carbajo et al., 2001) high temperature term is not dependent on stoichiometry and therefore the reduction of the thermal conductivity with increasing stoichiometry deviation, as predicted here, may be too conservative. However as shown below, the model presented here for urania can easily be adapted for thoria.

\subsection{Thoria}

The phonon contribution to the thermal conductivity is expressed by the same equation as for urania (Equation 4a) except that there is no dependence on stoichiometry deviation (x) for parameters A and B and the following values were proposed by Bakker et al. (1997): $\mathrm{A}=0.0042 \mathrm{mKW}^{-1}$ and $\mathrm{B}=0.000225 \mathrm{~mW}^{-1}$.

Belle and Berman (1984) it was pointed out that there are many uncertainties with evaluation of a radiative contribution to a thermal conductivity as it may depend on the relative mean free path and dimensions of the radiative objects' surface roughness that are not included in the original black body radiation model. The fuel is also not in equilibrium with the adjacent medium into which it is radiating energy. In the present evaluation we assume that the electromagnetic radiation in the thoria and urania fuels contains the same number of quantised electromagnetic waves at the same temperature and the only difference in their radiative conduction originates from the different critical angles of the total internal reflection on the surface of the fuel rods. Both urania and thoria have a higher refraction index (Szpunar and Szpunar, 2013b) than surrounding media, which has an index of refraction equal $\sim 1$. Siegel and Howell (2002) the effect of the total internal reflection of radiation on the surface of the body with a refraction index (n) larger than one and adjacent medium with an index of refraction equal to one has been evaluated. A reduction in the radiative heat conduction by $\mathrm{n}^{-2}$ was found. Using our previous first principles calculation of the index of refraction (Szpunar et al., 2013) the average ratio (wavelength between 200 and $7000 \mathrm{~nm}$ ) of the calculated index of refraction of thoria and urania was estimated as $S_{a v}$ equal to $\sim 0.6877$. Therefore we propose here to use Equation (5a-b) with the modification originating from the reduction of the total internal reflection due to lower index of refraction: $\mathrm{n}$ ( $\mathrm{n}_{\text {thoria }}=\mathrm{S}_{\mathrm{av}} \mathrm{n}_{\text {uarania }}$ ) of thoria by corresponding factor $\left(\mathrm{S}_{\mathrm{av}}\right)^{-2}=2.11447$ Equation (7):

$$
\mathrm{k}_{\mathrm{rad}}=16.05677 \times 10^{-7}\left(\alpha_{\mathrm{R}}(\mathrm{T})\right)^{-1} \mathrm{~T}^{3}
$$

where, the expression (Equation $5 b$ ) for $\alpha_{R}(T)$ is not changed. Since thoria has a reduced index of refraction, the respective radiative contribution is about twice that for urania in fuel rods.

In contrast it was evaluated that the electronic contribution is much smaller for thoria fuel. In the evaluation of the electronic contribution for thoria we set in Equation 6a-f, stoichiometric deviation (x) equal to 0 and increased $\Delta \mathrm{E}$, the electron mobility activation energy by a factor of three $\left(\Delta \mathrm{E}_{\text {thoria }}=3 \Delta \mathrm{E}_{\text {urania }}\right)$ as estimated from the ratio of the band gaps $(\sim 2 \mathrm{eV}$ for urania and $\sim 6$ $\mathrm{eV}$ for thoria (Szpunar et al., 2013; Szpunar and Szpunar, 2013b)). Since there is exponential dependence of the electronic contribution on the $\Delta \mathrm{E}$ (Equation $6 \mathrm{~b}$ ) the increase of the activation energy by a factor of three reduces drastically the electronic term.

\subsection{Thermal Conductivity of Thoria Versus Urania}

In Fig. 1 we compare the total thermal conductivity of thoria versus urania calculated as a sum of all contributions (phonons, electrons, radiation) Equation (8):

$\mathrm{k}=\mathrm{k}_{\mathrm{ph}}+\mathrm{k}_{\mathrm{e}}+\mathrm{k}_{\mathrm{rad}}$

We will be using here two previously proposed correlations for phonon contribution (Ellis et al., 2000; Lucuta et al., 1996) for a fully dense urania fuel (Equation $4 a-c)$ with the effect of stoichiometry deviation (x) included and with the electron and radiative contribution terms described by Equation 5-6. They are indicated in Fig. 1 by dot dot dash and broken line respectively and agree with each other well for stoichiometric fuel. Therefore we only plotted the phonon contribution for stoichiometric urania (triangles) and as indicated it is the only mechanism responsible for thermal conductivity at low temperature. Fink's (2000) correlation (indicated by solid line) was developed for $95 \%$ dense urania fuel without the dependence on $(\mathrm{x})$ and it is more conservative at lower temperature while slightly higher thermal conductivity is predicted at high temperatures.

The red dot dash curve represents the total conductivity of thoria, which is calculated as a sum of phonon contribution (Bakker et al., 1997), indicated by red triangles and polaron and radiative contribution as described above. It can readily be verified that the thermal conductivity of thoria is much higher at low temperatures than for urania and at high temperatures the phonon contribution decreases significantly, being only a few percent higher as evaluated in Table 2 for minimal conductivity ratios of thoria versus urania. 


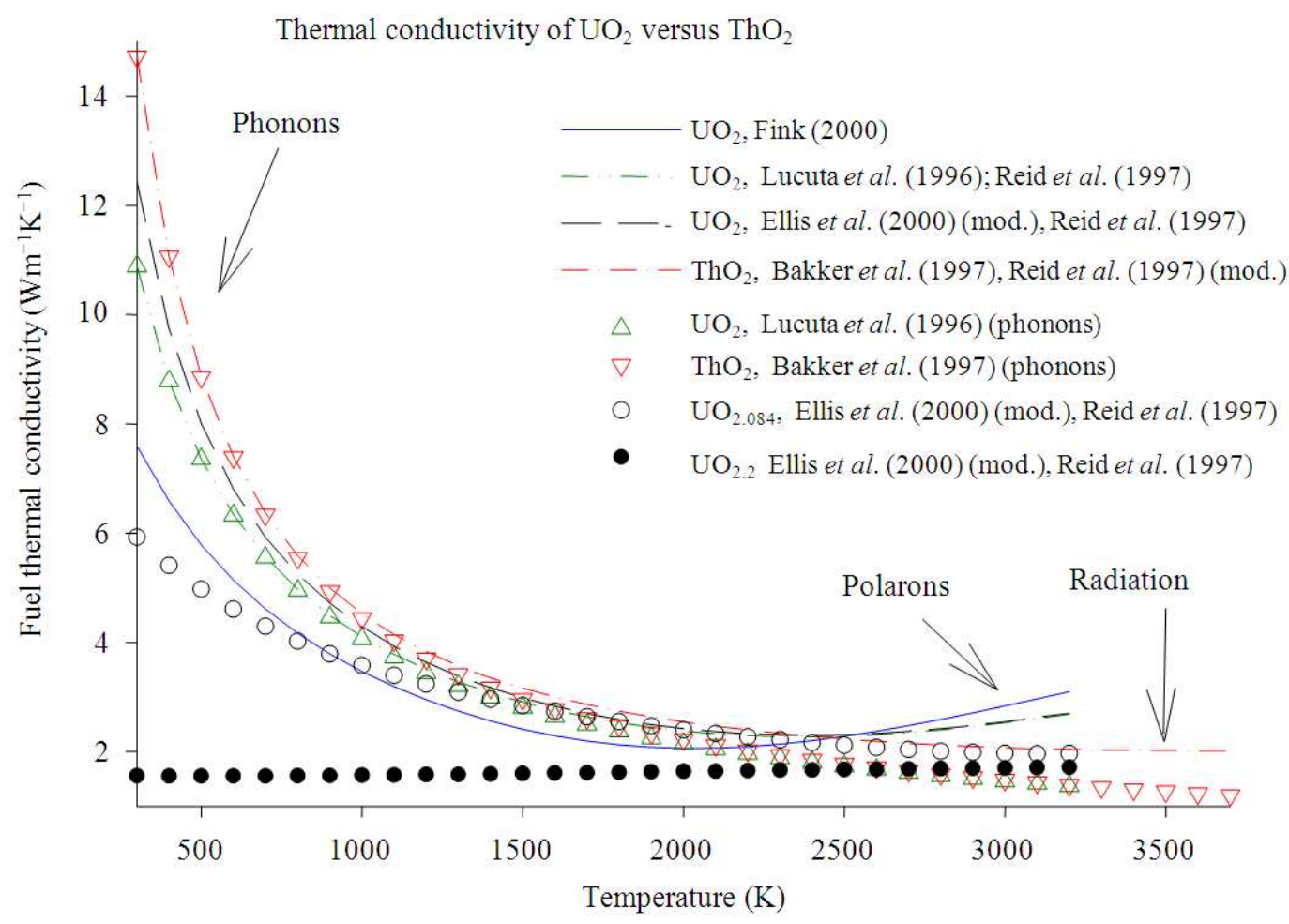

Fig. 1. The calculated thermal conductivity of urania and thoria as a function of temperature. The lines represent the total thermal conductivity (Equation 6) while triangles show phonon contribution (Equation 3a). The primary contribution at high temperature (electronic/polarons for urania and radiation for thoria). Various correlations as described in the text and indicated are used for the phonon contribution while other contributions are calculated using Eqs. 4-a except for the correlation recommended by Fink (2000) indicated by solid red curve. The circles show the reduced thermal con-ductivity of urania for stoichiometry deviation equal to 0.084 (open circles) and 0.2 (solid circles)

In Fig. 1 it is also illustrated that the thermal conductivity is significantly reduced for urania when oxidation $(\mathrm{x}=0.084$ : Open circles and $\mathrm{x}=0.2$ solid circles) takes place as also discussed before (Lewis et al., 2002).

In the presented model the thermal conductivity of urania at low stoichiometry deviation and high temperature originates primarily from electronic contribution as indicated in Fig. 1. In contrast for thoria, which has a radiative contribution about twice higher, the electronic contribution is negligible and therefore Fig. 1 indicates that the value of thoria conductivity at high temperature of around $2 \mathrm{Wm}^{-1} \mathrm{~K}^{-1}$ is radiative. This is in agreement with the value shown in figure 6.24 in (Belle and Berman, 1984) of the flat thermal conductivity of thoria at high temperature around $2 \mathrm{Wm}^{-1} \mathrm{~K}^{-1}$. The value of $2.33 \mathrm{Wm}^{-1} \mathrm{~K}^{-1}$ at $2300 \mathrm{~K}$ as shown in Fig. $\mathbf{1}$ is also in agreement with the $2.5 \mathrm{Wm}^{-1} \mathrm{~K}^{-1}$ value reported by Hyland (1983) where it is noted also that this thermal conductivity value is maintained up to melting temperature.

\subsection{The Temperature Profile in the Defective Fuel Rods}

Once the thermal conductivity is known one can calculate the temperature distribution in a cylindrical rod of urania (Lewis et al., 2002) or thoria fuel for a specified volumetric heat generation rate from fission (H) Equation (9):

$\mathrm{H}=\frac{\mathrm{P}}{\pi \mathrm{a}^{2}}\left[\frac{\left(\mathrm{aL}^{-1}\right)}{2 \mathrm{I}_{1}\left(\mathrm{aL}^{-1}\right)}\right] \mathrm{I}_{0}\left(\mathrm{rL}^{-1}\right)$

where, $\mathrm{P}$ is linear power $\left(\mathrm{P}=52 \mathrm{kWm}^{-1}\right)$ and "a" is the radius of the fuel rod $(\mathrm{a}=0.006 \mathrm{~m})$. The thermal neutron diffusion Length (L) equal to $1.1 \mathrm{~cm}$ for natural urania with the burnup of $8000 \mathrm{MWd} / \mathrm{t}$ as estimated in our previous work (Lewis et al., 2002) is also used here. The neutron diffusion length for pure thoria is longer but it will be shorter when doped with $\mathrm{Pu}$ or $\mathrm{U}$, therefore for simplicity we assume the same profile for the neutron 
flux depression (Equation 9) where $I_{0}$ and $I_{1}$ are the modified Bessel functions of the first kind.

The temperature profile in the nuclear fuel rod is calculated by solving the steady state heat conduction equation for cylindrical symmetry (Olander, 1976):

$$
\frac{1}{\mathrm{r}} \frac{\mathrm{d}}{\mathrm{dr}}\left(\mathrm{rk} \frac{\mathrm{dT}}{\mathrm{dr}}\right)+\mathrm{H}=0
$$

Equation (10a) is subject to the boundary conditions Equation (10b-10c):

$$
\begin{array}{ll}
\frac{\mathrm{dT}}{\mathrm{dr}}=0, & \mathrm{r}=0 \\
\mathrm{~T}=\mathrm{T}_{\mathrm{s}}, & \mathrm{r}=\mathrm{a}
\end{array}
$$

where, $T_{\mathrm{s}}$ is the fuel surface temperature, assumed here to be $870 \mathrm{~K}$ (Lewis et al., 2002). Here $\mathrm{k}$ is the thermal conductivity, which is a function of temperature and consequently depends on the radial position $r$. Additionally for operating defective nuclear fuel rods that are made from urania, fuel-oxidation may take place and therefore the thermal conductivity will be reduced with increasing stoichiometry deviation $(\mathrm{x})$ as discussed in the previous section and presented in Fig. 1. We assume here the time of origin of simulation to be the time after the zircalloy cladding has fully oxidised since, as calculated before (Szpunar et al., 2001), there is no significant urania fuel oxidation earlier due to a low value of oxygen potential in the presence of hydrogen originating from the oxidation of zirconium in steam. Recently fuel oxidation models in steam atmosphere were evaluated (Szpunar, 2012b) and while a more accurate equation for a steam dissociation was provided it was also demonstrated that there was not much effect on the calculated stoichiometry deviation; therefore we use here the simplified model used previously (Lewis et al., 2002; Szpunar et al., 2001). The estimate of equilibrium stoichiometry deviation (Blackburn, 1973) used here is conservative at high temperatures and the difference in the calculated equilibrium stoichiometry deviation at low temperatures from more conservative models is not significant (Szpunar and Szpunar, 2012).

In Fig. 2 the temperature profile in the fuel rod is shown as simulated after one day's irradiation time at a linear power of $52 \mathrm{kWm}-1$. When no oxidation takes place the predicted temperature profiles for urania and thoria are very similar (for the correlations used for thermal conductivity as indicated) and are presented by broken and solid lines for urania and a dot dash line for thoria in Fig. 2.

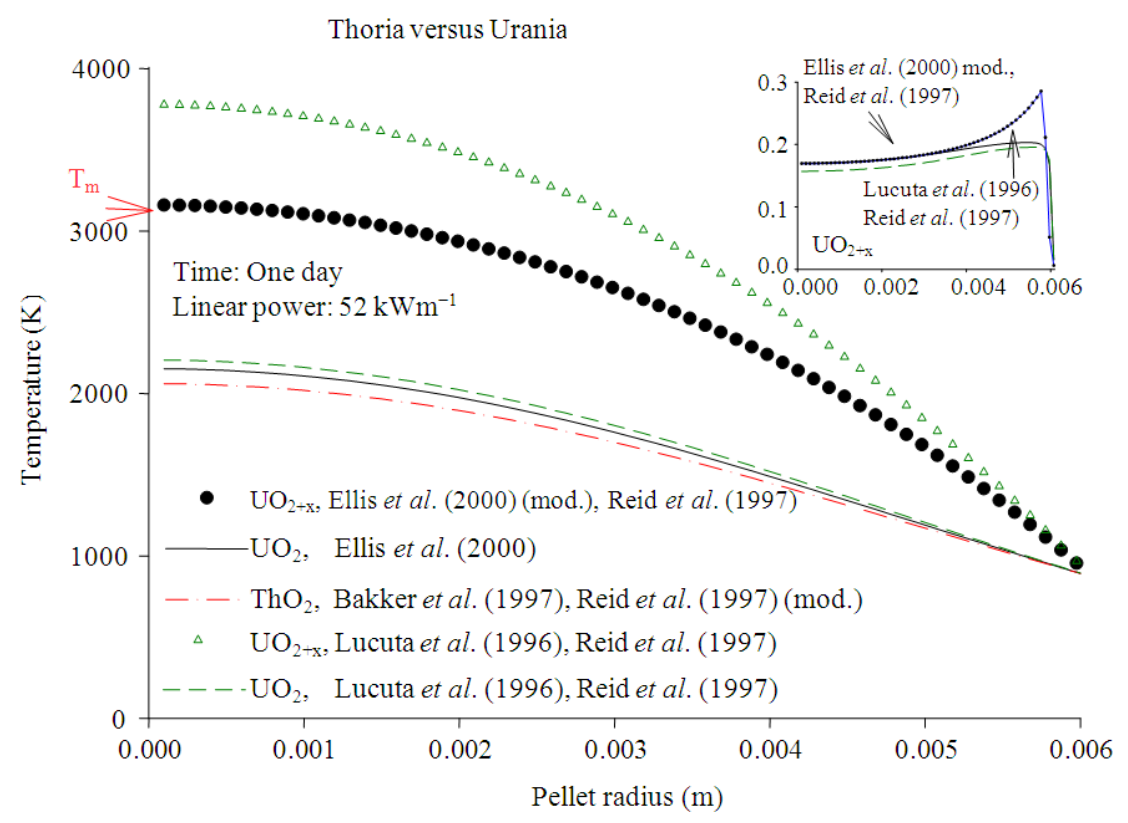

Fig, 2. Predicted fuel temperature profile for stoichiometric thoria and urania (indicated by line). The calculations in a defective fuel rod operating at a linear power of $52 \mathrm{~kW} / \mathrm{m}$ in which pure steam is present in the gap atmosphere are also shown and indicated by circles and triangles for two different correlations for thermal conductivity as indicated and described in the text. The arrow indicates the melting temperature (Tm) for urania: 3120K (Adamson et al., 1985). The temperature is drastically enhanced when urania fuel oxidation takes place and in the insert the respective stoichiometry deviations are shown as indicated. The pick close to the surface of the pellet is also shown for the results where the oxygen diffusion in the pellet was neglected (solid/dotted line in the insert) 


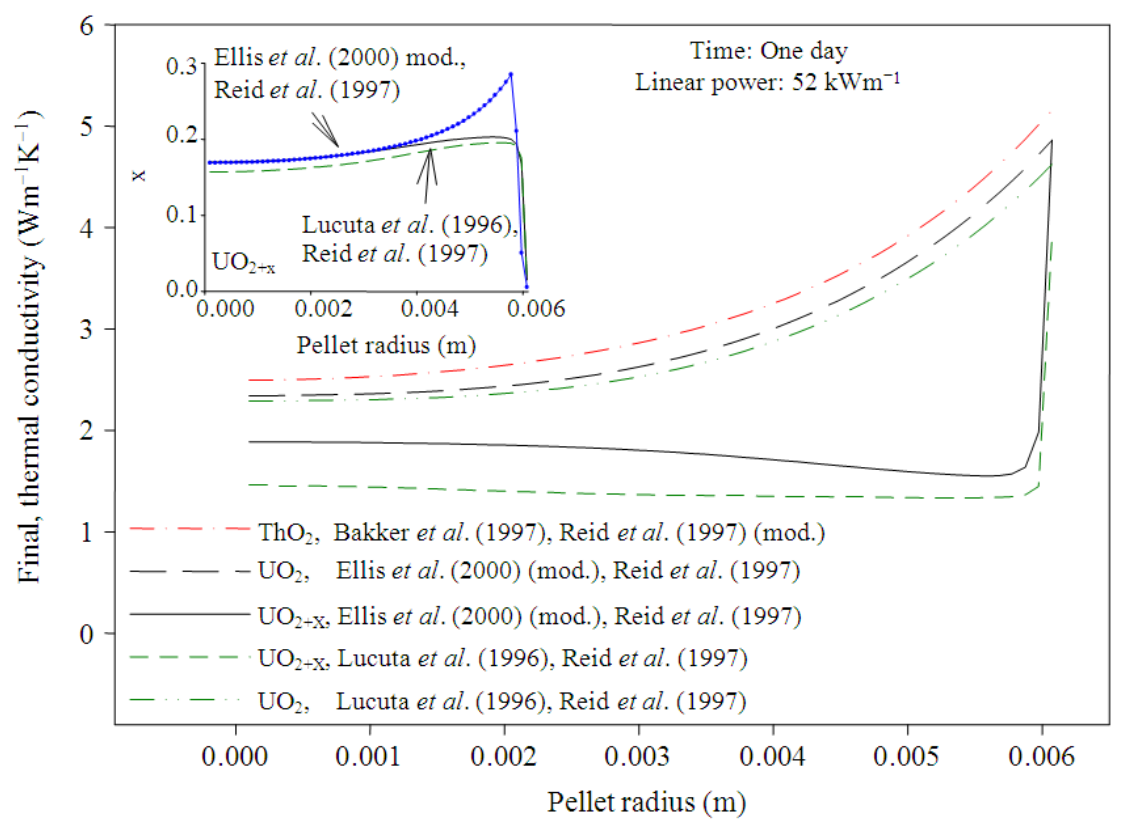

Fig. 3. The calculated thermal conductivity profile in urania and thoria pellet (as a function of the distance from the centre: $r$ ) after one day's irradiation at $52 \mathrm{kWm}-1$ linear power. The lines represent the total thermal conductivity using various correlations as described in the text and indicated. In the insert the respective stoichiometry deviations are shown as indicated. Additionally, indicated by a solid/dotted line with the pick close to the surface, the results for stoichiometry deviation are shown where oxygen diffusion in the pellet was neglected

However when urania fuel oxidation takes place the temperature is drastically increased (indicated by triangles and circles) and central melting in the fuel rod occurs. This can be compared with the melting temperature of urania $\left(\mathrm{T}_{\mathrm{m}}\right)$, indicated by the arrow, as observed experimentally (Lewis et al., 2002). This temperature increase is due to greatly reduced thermal conductivity as shown in Fig. 3. We also note that the correlation proposed by Carbajo et al. (2001) predicts an increase of temperature at the centre below melting $60 \mathrm{~K}$. In the inserts of Fig. 2 and 3 the calculated flat profile of the stoichiometry deviation of urania is also shown after one day. Additionally the dotted line shows that there would be a higher stoichiometry deviation close to the surface of the fuel if the diffusion of oxygen were not included as observed previously (Lewis et al., 2002).

\section{CONCLUSION}

It is important to include multidisciplinary tools in reactor safety analysis. First principles simulations are useful in assessing the behaviour of nuclear materials especially where there are no experimental data available. They are also useful as a complimentary tool in reactor safety analysis. Our analysis indicates that the main advantage of using thoria versus urania fuel is that it does not form oxides during accident and therefore there is no undesirable degradation of its thermal conductivity. The fuel melting observed in urania fuel rods during accident with steam ingress is not observed in thoria as its thermal conductivity remains high enough to dissipate excessive heat in the centre.

\section{ACKNOWLEDGEMENT}

The access to high performance supercomputers at CLUMEQ and Westgrid is acknowledged. The authors would like to acknowledge collaboration with B. Lewis during which the original version of the Maple code used here was developed and J. Ki-Seob Sim for collaboration on thoria fuel.

\section{REFERENCES}

Adamson, M.G., E.A. Aitken and R.W. Caputi, 1985. Experimental and thermodynamic evaluation of the melting behavior of irradiated oxide fuels. J. Nucl. Mater., 130: 349-365. DOI: 10.1016/00223115(85)90323-X 
Baer, Y. and J. Schoenes, 1980. Electronic structure and coulomb correlation energy in $\mathrm{UO}_{2}$ single crystal. Solid State Commun., 33: 885-888. DOI: 10.1016/0038-1098(80)91210-7

Bakker, K., E.H.P. Cordfunke, R.J.M. Konings and R.P.C. Schram, 1997. Critical evaluation of the thermal properties of $\mathrm{Th}_{2}$ and $\mathrm{Th} 1_{-\mathrm{y}} \mathrm{U}_{\mathrm{y}} \mathrm{O}_{2}$ and a survey of the literature data on $\mathrm{Th}_{1-\mathrm{y}} \mathrm{Pu}_{\mathrm{y}} \mathrm{O}_{2}$. J. Nucl. Mater., 250: 112. DOI: $10.1016 / \mathrm{S} 0022-3115(97) 00241-9$

Becke, A.D., 1993. A new mixing of hartree-fock and local density-functional theories. J. Chem. Phys., 98: 1372-1377. DOI: $10.1063 / 1.464304$

Belle, J. and R.M. Berman, 1984. Thorium dioxide: Properties and nuclear application. Naval Reactors Office, United States Department of Energy DOENE-0060, Washington, D.C.

Blackburn, P.E., 1973. Oxygen partial pressures over fast breeder reactor fuel, (I) a model for $\mathrm{UO}_{2 \pm \mathrm{x}}$. J. Nucl. Mater., 46: 244-252. DOI: 10.1016/00223115(73)90038-X

Cahill, D.G., S.K. Watson and R.O. Pohl, 1992. Lower limit to the thermal conductivity of disordered crystals. Phys. Rev. B, 46: 6131-6140. DOI: 10.1103/PhysRevB.46.6131

Carbajo, J.J., G.L. Yodera, S.G. Popov and V.K. Ivanov, 2001. A review of the thermophysical properties of MOX and $\mathrm{UO}_{2}$ fuels. J. Nucl. Mater., 288: 181-198. DOI: 10.1016/S0022-3115(01)00692-4

Ceperley, D.M. and B.J. Alder, 1980. Ground state of the electron gas by a stochastic method. Phys. Rev. Lett., 45: 566-569. DOI: 10.1103/PhysRevLett.45.566

Clausen, K., W. Hayes J.E. Macdonald, R. Osborn and P.G. Schnabel et al., 1987. Inelastic neutron scattering investigation of the lattice dynamics of $\mathrm{ThO}_{2}$ and $\mathrm{CeO}_{2}$. J. Chem. Soc., Faraday Trans., 283: 1109-1117. DOI: 10.1039/f29878301109

Cococcioni, M. and S. De Gironcoli, 2005. Linear response approach to the calculation of the effective interaction parameters in the LDA+U method. Phys. Rev. B, 71: 035105-1-035105-16. DOI: 10.1103/PhysRevB.71.035105

Ellis, W.E., J.D. Porter and T.L. Shaw, 2000. The effect of oxidation, Burnup and poisoning on the thermal conductivity of $\mathrm{UO}_{2}$ : A comparison of data with theory. Proceedings of the International Topical Meeting on Light Water Reactor Fuel Performance, Apr. 10-13, Park City, Utah, pp: 715-715.

Faber, Jr. J. and G.H. Lander, 1976. Neutron diffraction study of $\mathrm{UO}_{2}$ : Antiferromagnetic state. Phys. Rev. B, 14: 1151-1164. DOI: 10.1103/PhysRevB.14.1151
Fine, M.E., L.D. Brown and H.L. Marcus, 1984. Elastic constants versus melting temperature in metals. Scr. Metall., 18: 951-956. DOI: 10.1016/00369748(84)90267-9

Fink, J.K., 2000. Thermophysical properties of uranium dioxide. J. Nucl. Mater., 279: 1-18. DOI: 10.1016/S0022-3115(99)00273-1

Frazer, B.C., G. Shirane, D.E. Cox and C.E. Olsen, 1965. Neutron-diffraction study of antiferromagnetism in $\mathrm{UO}_{2}$. Phys. Rev., 140: A1448-A1452. DOI: 10.1103/PhysRev.140.A1448

Fritz, I.J., 1976. Elastic properties of $\mathrm{UO}_{2}$ at high pressure. J. Applied Phys., 47: 4353-4357. DOI: 10.1063/1.322438

Geng, H.Y., Y. Chen, Y. Kaneta and M. Kinoshita, 2007. Structural behavior of uranium dioxide under pressure by LSDA+U calculations. Phys. Rev. B, 75: 054111054118. DOI: 10.1103/PhysRevB.75.054111

Hyland, G.J., 1983. Thermal conductivity of solid UO2: Critique and recommendation. J. Nucl. Mater., 113: 125-132. DOI: 10.1016/0022-3115(83)90134-4

Iglesias, F.C., C.E.L. Hunt, F. Garisto and D.S. Cox, 1989. Ruthenium release kinetics from uranium oxides. Proceedings of the International Center for Heat and Mass Transfer Conference on Fission Product Transport Processes in Reactor Accidents, May 22-26, Dubrovnik, Yugoslavia, pp: 187-196.

Kohn, W. and L.J. Sham, 1965. Self-consistent equations including exchange and correlation effects. Phys. Rev., 140: A1133-A1138. DOI: 10.1103/PhysRev.140.A1133

Lander, G.H., 1980. Neutron scattering studies of the actinides. J. Magn. Magn. Mat., 15: 1208-1214. DOI: 10.1016/0304-8853(80)90257-7

Lewis, B.J., B. Szpunar and F.C. Iglesias, 2002. Fuel oxidation and thermal conductivity model for operating defective fuel rods. J. Nucl. Matter., 306: 30-43. DOI: 10.1016/S0022-3115(02)01231-X

Loopstra, O., 1970. The phase transition in $\alpha-\mathrm{U}_{3} \mathrm{O}_{8}$ at $210^{\circ} \mathrm{C}$. J. Appl. Cryst., 3: 94-96. DOI: 10.1107/S002188987000571X

Lucuta, P.G., H. Matzke and I.J. Hastings, 1996. A pragmatic approach to modelling thermal conductivity of irradiated $\mathrm{UO}_{2}$ fuel: Review and recommendations. J. Nucl. Mater., 232: 166-180. DOI: 10.1016/S0022-3115(96)00404-7

Macedo, P.M., W. Capps and J.B. Watchman, 1964. Elastic constants of single crystal $\mathrm{ThO}_{2}$ at $25^{\circ} \mathrm{C}$. J. Am. Ceram. Soc., 47: 651-661. DOI: 10.1111/j.1151-2916.1964.tb13130.x 
Mathews, M.D., B.R. Ambekar and A.K. Tyagi, 2000. Bulk and lattice thermal expansion of $\mathrm{Th}_{1-\mathrm{x}} \mathrm{Ce}_{\mathrm{x}} \mathrm{O}_{2}$. J. Nucl. Mater., 280: 246-249. DOI: 10.1016/S00223115(00)00068-4

McEachern R.J. and P. Taylor, 1998. A review of the oxidation of uranium dioxide at temperatures below $400^{\circ}$ C. J. Nucl. Mater., 254: 87-121. DOI: 10.1016/S0022-3115(97)00343-7

Olander, D.R., 1976. Fundamental Aspects of Nuclear Reactor Fuel Elements. 1st Edn., Technical Information Center, Oak Ridge, ISBN-10: 0870790315, pp: 612.

Perdew, J.P., K. Burke and M. Ernzerhof, 1966. Generalized gradient approximation made simple. Phys. Rev. Lett., 77: 3865-3868. DOI: 10.2172/7290222

Perdew, J.P., A. Ruzsinszky, G.I. Csonka, O.A. Vydrov and G.E. Scuseria, 2008. Restoring the densitygradient expansion for exchange in solids and surfaces. Phys. Rev. Lett., 100: 136406-136409. DOI: 10.1103/PhysRevLett.100.136406

Reid, P.J., M.J. Richards, F.C. Iglesias and A.C. Brito, 1997. SOURCE 2.0 model development: $\mathrm{UO}_{2}$ thermal properties. Proceedings of the 5th International Conference on CANDU Fuel, Sep. 2125, Toronto, pp: 321-341.

Ronchi, C. and J.P. Hiernaut, 1996. Experimental measurement of pre-melting and melting of thorium dioxide. J. Alloys Comp., 240: 179-185. DOI: 10.1016/0925-8388(96)02329-8

Sanati, M., R.C. Albers, T. Lookman and A. Saxena, 2011. Elastic constants, phonon density of states and thermal properties of $\mathrm{UO}_{2}$. Phys. Rev. B, 84: 014116014122. DOI: $10.1103 /$ PhysRevB.84.014116

Segall, M.D., P.L.D. Lindan, M.J. Probert, C.J. Pickard and P.J. Hasnip et al., 2002. First-principles simulation: Ideas, Illustrations and the CASTEP Code. J. Phys. Cond. Matt., 14: 2717-2743. DOI: 10.1088/0953-8984/14/11/301

Siegel, R. and J. Howell, 2002. Thermal Radiation Heat Transfer. 4th Edn., Taylor and Francis, New York, London, pp: 868.

Sullivan, J.D. and D.S. Cox, 1995. AECL progress in developing the DUPIC fuel fabrication process. Proceedings of the 4th International Confrence on CANDU Fuel, Oct. 1-4, Pembroke, Ontario, Canada, pp: 49-58.

Sviridova, A.A. and N.V. Suikovskaya, 1967. Transparence limits of interference films of hafnium and thorium oxides in the ultraviolet region of the spectrum. Opt. Spectrosc., 22: 509-509.
Szpunar, B. and J.A. Szpunar, 2012. Properties of recycled fuels; Density functional theory study. Proceedins of the 33rd Annual Conference of the Canadian Nuclear Society, Saskatoon, Canada, Jun. 10-13.

Szpunar, B., 2012a. Investigation of urania within LDA+U method. J. Phys. Chem. Solids, 73: 10031009. DOI: $10.1016 /$ j.jpcs.2012.03.007

Szpunar, B., 2012b. Assessment of the models of urania fuel oxidation in steam-rich atmosphere. J. Nucl. Sci. Technol., 49: 1186-1192. DOI: 10.1080/00223131.2012.739348

Szpunar, B., 2013. Multidisciplinary reactor safety studies: Application of first principles calculation. Proceedings of the 1st Annual International Conference on Physics and Chemistry, Jul. 22-25, Athens, Greece.

Szpunar, B., B.J. Lewis, V.I. Arimescu, R.S. Dickson and L.W. Dickson, 2001. Three-component gas mixture transport in defective candu fuel rods. J. Nucl. Mater., 294: 315-329. DOI: 10.1016/S0022-3115(01)00422-6

Szpunar, B. and J.A. Szpunar, 2004. The crystal structure of pure and doped urania. Proceedings of the International Conference on Simulation Methods in Nuclear Engineering, Canadian Nuclear Society, Montreal, Oct. 13-15, pp: 36-36.

Szpunar, B. and J.A. Szpunar, 2013a. Application of density functional theory in assessing properties of thoria and recycled fuels. J. Nucl. Mater., 439: 243250. DOI: 10.1016/j.jnucmat.2012.10.009.

Szpunar, B. and J.A. Szpunar, 2013b. Density functional studies of selected metal dioxides. J. Phys. Chem. Solids, 74: 1632-1639. DOI: 10.1016/j.jpcs.2013.06.007

Szpunar, B., J.A. Szpunar, V. Milman and A. Goldberg, 2013. Implication of volume changes in uranium oxides: A density functional study. Solid State Sci., 24: 44-63. DOI: 10.1016/j.solidstatesciences.2013.06.013

Wilkins, S.B., R. Caciuffo, C. Detlefs, J. Rebizant and E. Colineau et al., 2006. Direct observation of electricquadrupolar order in $\mathrm{UO}_{2}$. Phys. Rev. B, 73: 060406060409. DOI: 10.1103/PhysRevB.73.060406

Wu, A. and R.E. Cohen, 2006. More accurate generalized gradient approximation for solids. Phys. Rev. B, 73: 235116-235121. DOI: 10.1103/PhysRevB.73.235116

Wyckoff, R.W.G., 1963. Crystal Structures. 2nd Edn., John Wiley and Sons, Inc., New York, pp: 407.

Yun, Y., J. Rusz, M.T. Suzuki and P.M. Oppeneer, 2011. First-principles investigation of higher oxides of uranium and neptunium: $\mathrm{U}_{3} \mathrm{O}_{8}$ and $\mathrm{Np}_{2} \mathrm{O}_{5}$. Phys. Rev. B, 83: 075109-075118. DOI: 10.1103/PhysRevB.83.075109 